\title{
When Does Competition Lead to Efficient
}

\section{Investments?}

\author{
Kalyan Chatterjee and Y. Stephen Chiu*
}

October 28, 1999

\footnotetext{
Chatterjee: Department of Management Science and Information Sciences and Department of Economics, Pennsylvania State University, University Park PA 16802, USA. Chiu: Department of Decision Sciences and Managerial Economics, The Chinese University of Hong Kong, Shatin, HONG KONG, email:ywchiu@baf.msmail.cuhk.edu.hk. The two authors are grateful to seminar participants at The Chinese University of Hong Kong and the University of Hong Kong for comments on earlier versions of the paper. Part of the research was performed when each author was visiting the other author's institution. Hospitality provided by the two institutions are acknowledged. The two authors are responsible for any remaining errors. For correspondence, please direct to Chiu.
} 


\begin{abstract}
The paper studies agents' investment decisions between general and specific investments under different ownership structures in a thin, decentralized market where each agent's decision affects the decisions and welfare of other (otherwise unrelated) agents mainly through indirect market linkages. The paper demonstrates that "excess competition among investors," in every equilibrium, will lead to efficient investments, regardless of asset ownership. In the absence of such excess competition, in every equilibrium, inefficient investments will result, unless some special ownership arrangement is made. The problem in which the choice variable is investment level, instead of investment type, is also studied.
\end{abstract}




\section{Introduction}

In this paper, we study agents' investment decisions under different ownership structures in a thin, decentralized market where each agent's decision affects the decisions and welfare of other (otherwise unrelated) agents mainly through indirect market linkages. Since the seminal papers by Grossman and Hart (1986) and Hart and Moore (1990), several papers have been written to examine the costs and benefits of integration in the same incomplete contracting, or property rights approach, ${ }^{1}$ but under various, different assumptions. ${ }^{2}$ Most of these analyses, including Grossman and Hart and Hart and Moore, confine themselves to the study of the incentive issues among agents within a firm or within a bilateral relationship, while taking the outside market as given. The present paper is an attempt to expand the domain of study to an explicitly endogenized market environment.

The following example is illustrative of the problem which the present paper plans to study. An economy consists of a number of bookkeepers and clients. A bookkeeper is supposed to make human capital investment in order to assist a client. She can choose either a general investment, which enables her to help any client, or a specific investment, which while yielding a greater surplus enables her to help some preidentified client only. In order to make an investment, each bookkeeper needs to have access to a computer. In order to use a bookkeeper, each client needs to have

\footnotetext{
${ }^{1}$ For a nontechnical introduction of the approach, see Hart (1995).

${ }^{2}$ See, e.g., Chiu (1998), Dasgupta and Tao (1999), de Meza and Lockwood (1998a,b), and Rajan and Zingales (1998).
} 
access to both a computer and a printer. We are interested in three types of simple ownership structures: (1) the bookkeeper owns both a computer and a printer and hires the "client" to manage the operations once the human capital investment is made; (2) the bookkeeper owns a computer and the client a printer; and (3) the client owns both a computer and a printer and hires the bookkeeper to work for him. Suppose agents are organized in these three types of ownership structures. We want to know: what investment decisions will bookkeepers of different ownership structures make? More abstractly, the paper looks at a two stage game in which sellers make investment decisions in the first stage, and bargain with buyers in the second stage. As in the example, what a seller needs to choose is the investment type, rather than the investment level, which is commonly studied in the literature. To help make a contrast, we also provide analysis on the case where the choice is the investment level.

There are two reasons why we think the proposed problem is interesting. First, choice of investment type, which is relatively neglected in the property rights literature, is a frequent decision which firms need to make. It turns out that the roles of competition and asset ownership in affecting investment decisions are quite different between problems with choice of investment types and those with investment levels. The second point is related to the so called adverse effect of ownership - that owning more assets reduces the owner's investment incentives. It has been pointed out that availability of alternative buyers may motivate a seller to make general investments (see, e.g., Tirole 1999, and Segal and Whinston 1998). This observation, however, is incomplete in the sense that the seller may also face competition from other counterpart sellers, or the alternative buyers have other alternatives as well. Hence to study 
an agent's investment decision, we need to embed this problem in a larger one where agents interact through the market.

In the literature, the adverse effect of ownership has been the focus of study in Chiu (1998), de Meza and Lockwood (1998a), and Rajan and Zingales (1998), among others. ${ }^{3}$ Chiu and de Meza and Lockwood argue for the possibility that assigning more assets to an agent may lead to a lower investment by that agent when strategic bargaining honoring the "outside option principle" is used. In addition to strategic bargaining, Rajan and Zingales provide two other instances - namely that choice variable is investment type, and that investment reduces the investor's outside value -in which they maintain the effect will take place. All these analyses, however, focus on the relationship within a firm, and do not consider the interplay between market competition and investment.

The main results of the paper are as follows. First, when there are more sellers than buyers, in every equilibrium all investing sellers will make efficient, specific investments, regardless of asset ownership; when there are fewer sellers than buyers, in every equilibrium both employer-sellers and independent sellers will make inefficient, general investments, while employee-sellers will make efficient, specific investments. Second, ownership may affect the probability of trade, in addition to the investment choice. When there are more sellers than buyers, there will be multiple equilibria in the investment game. Sellers who own both assets will trade with certainty in every equilibrium, and sellers with one or no asset may not have a trade in some equilib-

\footnotetext{
${ }^{3}$ It has been touched on earlier by Holmstrom and Tirole (1991) and Tirole. Maskin and Tirole (1999) also argues for the optimality of non-simple asset ownership.
} 
rium. ${ }^{4}$ Third, the role of competition and ownership in the extension of the model where the choice is investment level is quite different. There exists an equilibrium with the following properties. When the investment is specific enough (outside option does not increase rapidly enough, or even decreases with the investment level), both ownership and competition play no role in affecting investment choices. When the investment is relatively more general, employer-sellers will make a higher level and more efficient investment than independent and employee-sellers do in the presence of excess competition among buyers, and make the same investment as those other sellers do in the absence of excess competition among buyers.

The first result, while demonstrating the harmful effect of ownership, highlights the role of excess competition among investors in enhancing efficiency. ${ }^{5}$ Note that our results are invariant in all equilibria; while the features we identify can conceivably be exhibited as an equilibrium outcome, it is not obvious that they are in fact invariant properties of all equilibria. An implication of the role of excess competition identified above is that when the asset is not transferable, easier entry to the sellers market may be the only way to remedy otherwise possible inefficiency.

A comparison between the first and the third results shows that there is a sharp

\footnotetext{
${ }^{4}$ Bolton and Whinston (1993) consider the case of an upstream firm (seller) and two downstream firms (buyers), where the buyers make investments. They exogenously specify a probability that the seller will not be able to serve both buyers. Their paper does not deal with general versus specific investment choices, and their results are, not surprisingly, very different from ours. However, their paper is one of the earliest we know of to pose the issue of investment and ownership in a market context.

${ }^{5}$ This can be understood through the following "stylized" fact which we think common in US graduate schools. When there are a large number of available supervisors to choose from, a graduate student is better off by investing more in general knowledge without hurrying in sticking to a particular teacher. When there is a limited number of available supervisors, he or she is better off by earlier engagement with a particular teacher and hence earlier investment that is specific to that teacher.
} 
distinction between the incentives associated with investment type and with investment level. Our result that adverse effect of ownership does not exist even if an increase in investment can lead to a decrease in outside option contrasts with that of Rajan and Zingales. The contribution here is not to point out the general possibility of the adverse effect of ownership but to clarify the conditions for such an effect to take place. Since the apparent possibility of contradictory results is sometimes viewed as one difficulty of the property rights approach (see Holmstrom 1999), we think such clarification is needed.

As far as we know, the only paper that studies investment decisions in a market context in the property rights literature is the paper by de Meza and Lockwood (1998b). The paper, however, differs significantly from ours. Among other differences, it adopts a search approach, which makes each agent in the model atomistic and nonpivotal, and a repeated production framework, which differs from most papers in the literature, including ours, where a single period problem is studied.

The rest of the paper is organized as follows. Section 2 presents the model. Section 3 presents our bargaining solution as well as justifications. Section 4 studies the investment choice given the bargaining solution introduced in Section 3. Section 5 provides two extensions, one involving choices of investment levels, and the other involving choices of investment types. Section 6 contains some concluding remarks.

\section{Model}

There are two given sets of agents, whom we call sellers and buyers, respectively. Denote the set of each as $S$ and $B$, respectively. There are two kinds of assets, $a_{s}$ 
and $a_{b}$. There are equal numbers of $a_{s}$ assets and sellers, and equal numbers of $a_{b}$ assets and buyers. The assets are important because each seller needs to have access to a piece of $a_{s}$ in order to make and transfer any investment, and each buyer needs to have access to a piece of $a_{b}$ in order to make use of the investment made by and received from any seller.

We are interested in a market consisting of three types of firms. A type 1 firm is an integrated firm in which a seller owns a pair of $a_{s}$ and $a_{b}$, and hires a buyer without any asset to manage her $a_{b}$; the set of these sellers and buyers is denoted by $S^{1}$ and $B^{1}$, respectively. A type 2 firm is an independent seller or buyer who owns one piece of $a_{s}$ or $a_{b}$, respectively; the set of these sellers and buyers is denoted by $S^{2}$ and $B^{2}$, respectively. A type 3 firm is an integrated firm in which a buyer owns a pair of $a_{s}$ and $a_{b}$, and hires a seller without any asset to manage his $a_{s}$; the set of these sellers and buyers is denoted by $S^{3}$ and $B^{3}$, respectively. Clearly, $S=S^{1} \cup S^{2} \cup S^{3}$, $B=B^{1} \cup B^{2} \cup B^{3}, S^{i} \cap S^{j}=\phi$, for $i \neq j$ and $B^{i} \cap B^{j}=\phi$ for $i \neq j$. Denoting $|A|$ as the cardinality of set $A$, we have $\left|S^{1}\right|=\left|B^{1}\right|,\left|S^{3}\right|=\left|B^{3}\right|,|S|=\left|S^{1}\right|+\left|S^{2}\right|+\left|S^{3}\right|$, and $|B|=\left|B^{1}\right|+\left|B^{2}\right|+\left|B^{3}\right|$.

Given the market structure, we consider a two stage game in which sellers each make investments simultaneously in stage 1 , and bargain and trade with buyers in stage 2. Each seller can make either a general investment or a specific investment. A general investment yields a valuation of $m>0$ to one who purchases it. A specific investment yields a valuation of $M>m>M / 2$ to the buyer for whom the investment is specifically made, and yields a zero valuation otherwise. To make an investment, a seller incurs the same cost $c>0$, regardless of the seller's identity and the investment 
type. To be economically interesting, we assume that $M / 2-c>m / 2-c>0$. Clearly, a specific investment is more efficient than a general investment, as long as it does not lead to duplication. We also assume that each seller's investment can be used by at most one buyer, that investments are non-resalable, and that each buyer will need only one investment. We assume also that the terms of trade are determined by ex post bargaining, which will be explained in detail in the next section. To be consistent with the property rights literature, we adopt the assumption that investments, exchange, and bargaining are voluntary and hence integration per se does not change the available investment types and parties with whom the bargaining is conducted. All of the above, including the investment decisions once made and the bargaining solution, are commonly known.

\section{Preliminaries}

In this section, we first introduce more notation for stage 2 bargaining and then describe the bargaining solution to be used. Consider a partition of $S^{i}, i=1,2,3$. Let $S_{1}^{i} \subseteq S^{i}$ be the set of sellers with a general investment; $S_{2}^{i} \subseteq S^{i}$ the set of sellers in $S^{i}$ with a specific investment for a buyer in $B^{l}, l \geq i$ such that no other seller in $S^{r}, r \leq l$ has the same investment (i.e., nonduplicate specific investment) ${ }^{6} S_{3}^{i} \subseteq S^{i}$ the set of sellers with a specific investment for some buyer in $B^{l}, l \geq i$ such that there is some other seller in $S^{r}, r \leq l$ who also has the same investment (i.e., duplicate

\footnotetext{
${ }^{6}$ Hereafter, we will simply refer a seller's investment in such a scenario as a nonduplicate specific investment.
} 
specific investment) $;^{7} S_{4}^{i} \subseteq S^{i}$ the set of sellers in $S^{i}$ with a specific investment which is not specific to anybody in $B^{l}, l \geq i$; and $S_{5}^{i} \subseteq S^{i}$ the set of sellers in $S^{i}$ with no investments at all. Similarly, consider a partition of $B^{i}, i=1,2,3$. Let $B_{1}^{i} \subseteq$ $B^{i}$ be the set of buyers for whom no sellers in $S^{r}, r \leq i$ have specific investments; $B_{2}^{i} \subseteq B^{i}$ the set of buyers for whom there is only one seller in $S^{r}, r \leq i$ that has a specific investment, and $B_{3}^{i} \subseteq B^{i}$ the set of buyers for whom there are more than one seller in $\cup_{r \leq i} S^{r}$ that have specific investments. ${ }^{8}$ Clearly, $\sum_{j=1}^{5}\left|S_{j}^{i}\right|=\left|S^{i}\right|$ and $\sum_{j=1}^{3}\left|B_{j}^{i}\right|=\left|B^{i}\right|, i=1,2,3$. It is straightforward to check that

$$
\sum_{i=1}^{3}\left|S_{2}^{i}\right|=\sum_{i=1}^{3}\left|B_{2}^{i}\right| .
$$

Defining $\Delta^{i} \equiv\left|S_{1}^{i}\right|-\left|B_{1}^{i}\right|$ as the excess supply of general investments from $S^{i}$ to $B^{i}, i=1,2,3$, we can define

$$
\begin{aligned}
E^{1} & \equiv \Delta^{1}+\min \left\{0, \Delta^{2}+\min \left\{0, \Delta^{3}\right\}\right\}, \\
E^{2} & \equiv \max \left\{0, \Delta^{1}\right\}+\Delta^{2}+\min \left\{0, \Delta^{3}\right\}, \\
\text { and } \quad E^{3} & \equiv \max \left\{0, \max \left\{0, \Delta^{1}\right\}+\Delta^{2}\right\}+\Delta^{3}
\end{aligned}
$$

as the excess supply of general investments (from $S^{1} \cup S^{2} \cup S^{3}$ ) to buyers in $B^{i}, i=$

\footnotetext{
${ }^{7}$ Hereafter, we will simply refer a seller's investment in such a scenario as a duplicate specific investment.

${ }^{8}$ Since members in $S_{4}^{i}$ and $S_{5}^{i}$ do not play any role in the bargaining process, we will simply ignore them in the later-on analysis, nor will we explicitly specify their payoffs in the description of the bargaining solution.
} 
$1,2,3$, respectively. Let us consider one of them, say $E^{1}$, a bit more. Since buyers in $B_{1}^{1}$ do not own any $a_{s}$ and $a_{b}$, they can obtain general investments only by purchasing from sellers who have both $a_{s}$ and $a_{b}$, i.e., sellers in $S_{1}^{1}$, and not from anybody else. Hence even if $\Delta^{2} \geq 0$ and $\Delta^{3} \geq 0$, the excess supply of general investment to $B^{1}$ is simply $\left|S_{1}^{1}\right|-\left|B_{1}^{1}\right|$, i.e., $\Delta^{1}$. However, buyers in $B_{1}^{2}$ and $B_{1}^{3}$ may also need to buy from $S_{1}^{1}$ in the cases that $\Delta^{2}<0$ or $\Delta^{3}<0$. Hence taking this possibility into account, the excess supply of general investments to buyers in $B^{1}$ is further reduced, as described in (3.2). Similarly, $E^{2}$ and $E^{3}$ are defined. ${ }^{9}$

\subsection{Bargaining Solution}

Let $p_{1}^{i}, p_{2}^{i}$, and $p_{3}^{i}$ be the equilibrium price in trade obtained by sellers in $S_{1}^{i}$ (general investments), $S_{2}^{i}$ (nonduplicate specific investments), and $S_{3}^{i}$ (duplicate specific investments), respectively. Explicitly, we assume an equal treatment principle that sellers who made similar investment decisions receive the same payoff. Throughout the paper, we will make use of a bargaining solution with the following properties:

1. Matching between sellers and buyers is efficient, i.e., matching is such that social welfare is maximized, and

2. The equilibrium trading prices $p_{j}^{i}$ are as described in Table 1.

\footnotetext{
${ }^{9} \mathrm{~A}$ little thought shows that $\Delta^{1}$ must be nonpositive. Hence the first term in the right hand side of $(3.3)-\max \left\{0, \Delta^{1}\right\}-$ is zero and can be omitted from the expression. But we choose to retain the expression in (3.3) so that the parallel structures of $E^{1}, E^{2}$, and $E^{3}$ are more apparent.
} 


\begin{tabular}{|l|l|l|l|}
\hline$E^{i}$ & $p_{1}^{i}$ & $p_{2}^{i}$ & $p_{3}^{i}$ \\
\hline$>0$ & 0 & $M-m$ & 0 \\
\hline$=0$ & $m / 2$ & $M / 2$ & 0 \\
\hline$<0$ & $m$ & $M / 2$ & 0 \\
\hline
\end{tabular}

Table 1.

Remark 1. It is routine to check that $E^{1} \leq E^{2} \leq E^{3}$, implying that $p_{1}^{1} \geq p_{1}^{2} \geq p_{1}^{3}$.

Consider the payoff from making a general investment. When there is zero excess supply of general investments to buyers in $B^{i}\left(E^{i}=0\right)$, the ex post surplus of a general investment $m$ is equally split between its seller and buyer, i.e., a result of NashRubinstein bargaining outcome prevails. ${ }^{10}$ When there is excess demand $\left(E^{i}<0\right)$ or excess supply $\left(E^{i}>0\right)$, the price is driven up to clear the market (at a price of $m$ ), or down to clear the market (at a price of 0 ), resulting in a gain of $m$ or 0 to the seller, respectively. This illustrates a feature of the auction-Walrasian outcome. Note that making a general investment is not necessarily more secure than a specific investment, even though there may be a sizeable number of alternative buyers for general investments in the market.

Consider next the payoff from making a specific investment. It is clear that the payoff depends very much on whether or not the specific investment is duplicated. In case of duplication, competition among sellers with the same specific investment

\footnotetext{
${ }^{10}$ See Nash (1950) and Rubinstein (1982) for the Nash bargaining solution and Rubinstein alternating bargaining game, respectively.
} 
leads to a zero price; the buyer extracts all of the surplus created by the specific investment. This again illustrates the auction-Walrasian outcome. When the specific investment is distinct, the price depends very much on whether or not the outside option is binding. The outside option that buyers in $B_{2}^{i}, i=1,2,3$ can take is a general investment. When there is excess supply of general investment (i.e., $E^{i}>0$ ), a general investment is sold at a price of 0 , resulting in a benefit of $m$ to a buyer. Therefore a buyer in $B_{2}^{i}, i=1,2,3$, can secure a payoff of $\max \{m, M / 2\}$, resulting in the nonduplicate specific investment's price equaling $\min \{M-m, M / 2\}$. By our earlier assumption that $m>M / 2$, this price becomes $M-m$. When there is no excess supply (i.e., $E^{i} \leq 0$ ), purchasing a general investment (at a price of $m / 2$ or $m$ ) will bring in a benefit of at most $m / 2$. Therefore, a buyer in $B_{2}^{i}$ will consider this outside option unattractive, and the distinct investment's price is $M / 2, i=1,2,3$. This is a generalization of the outside option principle in 2 person noncooperative bargaining. ${ }^{11}$

\subsection{Justification of the Bargaining Solution}

Our bargaining solution is an equilibrium in the following generalized alternating bargaining game a la Rubinstein (1982). The post-investment bargaining stage consists possibly of an infinite number of substages $t=1,2,3, \ldots$ In odd substages, each seller makes public offers on how much she is willing to accept to sell her investment. It is assumed that each offer is publicly announced, and available to any responder at the same, nonpreferential price. Each buyer publicly announces whether and from whom

\footnotetext{
${ }^{11}$ See Binmore, Rubinstein, and Wolinsky (1986) and Sutton (1986) for the outside option principle, and Binmore, Shaked, and Sutton (1989) for experimental support of the principle.
} 
he is willing to accept an offer; each buyer can accept at most one offer. If an offer is accepted by one responder, then the proposer and the responder trade accordingly. If it is accepted by more than one responder, then among the acceptors one is randomly chosen with equal probability for the trading. Players who have traded will disappear from the game, the remaining players will proceed to the next substage to repeat the preceding substage with the roles of proposers and responders being switched. The process goes on until all opportunities of potentially beneficial trade are exhausted. Agents have the same common discount factor $\delta<1$ across substages. The structure and payoff of the game are commonly known.

The proof that this game gives our bargaining solution as one limiting equilibrium outcome as $\delta$ approaches unity is fairly tedious. A sketch is relegated to the Appendix. Two remarks on the assumptions of the bargaining game are in order. First, we assume that each offer is publicly announced and available to any responder at the same, nonpreferential price. This restriction is made to simplify the exposition, and there is no particular reason why offers cannot have other properties. In fact, one can show that the equilibrium outcome that we are interested in survives the relaxation that offers are allowed to be also private. Since we do not claim uniqueness of the equilibrium outcome here, we do not see any serious problem with this assumption. Second, given the structure of the bargaining game, collusion among sellers or among buyers is explicitly ruled out in the model. When collusion among sellers or among buyers is allowed (e.g., the Shapley value is used), the long side's agents' payoffs from general investment may not drop to zero.

This latter consideration does not, however, have as much force as it first appears 
to have. The reason is that such a collusion is not immune to deviation. To see this, consider a simple economy in which all firms are independent, there are more sellers than buyers, and all sellers make general investment. Since the quantity supplied of general investment is greater than the quantity demanded, our bargaining solution predicts that all general investments that are sold will be sold at a zero price. Suppose this is not the case, and some seller $s^{\prime}$ sells her general investment at a positive price $p>0$. All other agents then receive a total payoff of at most $n m-p<n m$. But by colluding to form a subcoalition, these other agents jointly obtain $\mathrm{nm}$. Hence there must exist some new allocation that makes each of them better off. In other words, collusion among agents on the long side will be broken by other coalitions. More important, any allocation that is in the core must have the property that players on the long side receive a zero payoff. ${ }^{12}$ The following Lemma is easy to show (all proofs of lemmas and propositions are relegated to the Appendix).

Lemma 3.1. 1. The allocation resulting from our bargaining solution is in the core.

2. If an allocation is in the core, then it must prescribe that:

(a) efficient matching, i.e., matching is such that social welfare is maximized.

(b) for $E^{i}>0, p_{1}^{i}=0, i=1,2,3$.

(c) for $E^{i}<0, p_{1}^{i}=m, i=1,2,3$.

(d) $p_{3}^{i}=0, i=1,2,3$.

\footnotetext{
${ }^{12}$ In other words, the allocation given by the Shapley value, which is used by Hart and Moore and Rajan and Zingales, is not in the core, despite the core's existence. See Shapley (1955), Gul (1989, 1999), and Hart and Mas-Colell (1996) for the foundations of the Shapley value.
} 
(e) $p_{1}^{i}=0 \Rightarrow p_{2}^{i} \leq M-m$.

Note that two other conditions are required to exactly tie down our bargaining solution; namely (1) the equal split of the surplus when the outside option is nonbinding, and (2) the payoff a player receives from a bilateral relationship simply matches his/her outside option if the outside option is binding. Since these two features of our bargaining solution are less controversial and since it is reasonable to require that the bargaining solution be in the core, our bargaining solution appears a reasonable one.

\section{Investment Decisions}

In this Section, we study the investment game using the bargaining solution previously stipulated. It turns out that the result will depend critically on whether there are more sellers than buyers.

\subsection{Excess Competition among Sellers $(|S| \geq|B|)$}

We first study the case in which $|S| \geq|B|$. The following result is easy to obtain (proof omitted).

Proposition 4.1. Suppose $|S| \geq|B|$. The following is an equilibrium outcome:

1. All employer-sellers (sellers in $S^{1}$ ) make investments specific to their respective employee-buyers.

2. $\left|B^{2}\right|$ independent sellers (sellers in $S^{2}$ ) make investments specific to a different independent buyer. 
3. All employee-sellers (sellers in $S^{3}$ ) make investments specific to their respective employer-buyers.

4. Every specific investment is traded at a price of $M / 2$, i.e., the ex post gain from trade is equally split between a seller and a buyer.

That the above is an equilibrium outcome is easy to understand. Consider an employer-seller in $S^{1}$. Given the investment choices of all other sellers as prescribed by Proposition 3.1, by making a specific investment, the seller basically faces a bilateral monopoly with her employee-buyer. This leads to an equal split of ex post surplus $M$. By making general investment, she still faces a bilateral monopoly with her employeebuyer, and hence gets only $m / 2$ from bargaining. It is true that her general investment is available to all other buyers. But this apparent availability of more buyers does not enhance her bargaining power. The essence is that each other buyer has a specific investment available for him, and that the specific investment yields a greater benefit to that buyer than a general investment does. As a result, the general investment that this employer-seller has is unattractive to other buyers, and hence making such an investment is not a wise decision. By a similar argument, the specific investment prescriptions for $S^{2}$ and $S^{3}$ are also optimal.

Note that some independent sellers do not make any investment, given that all sellers in $S^{1}$ and $S^{3}$ and $\left|B^{2}\right|$ independent sellers in $S^{2}$ have specific investments. Let us consider the optimal strategy of one of these noninvesting sellers (the seller must be in $S^{2}$ ). Suppose all other sellers follow the prescription above. By making a general investment, she has a group of potential buyers (all buyers in $B^{2}$ and $B^{3}$ ). 
The difficulty is that all of these buyers already have a specific investment available in the market. The effect of the availability of the general investment is simply to reduce the equilibrium price of these specific investments from $M / 2$ to $M-m$, while the general investment will never be purchased. On the other hand, if the seller makes a specific investment for some buyer in $B^{2}$ and $B^{3}$ (any buyer in $B^{1}$ will not be able to purchase from sellers in $S^{2}$ ), then the investment becomes duplicated and will not be sold at any positive price. Hence no investment is indeed optimal for the seller.

Proposition 4.2. Suppose $|S| \geq|B|$. Every equilibrium outcome of the investment game has the following properties:

1. Only $|B|$ distinct specific investments are made; no general investments are made.

2. For every buyer, an investment specific for him will be produced.

3. Employer-sellers (sellers in $S^{1}$ ) each will make a specific investment.

4. The specific investments that employer-sellers (employee-sellers) made are for employee-buyers (employer-buyers).

5. The specific investments that independent sellers (sellers in $S^{2}$ ) made are for independent buyers (buyers in $B^{2}$ ) and possibly for employer-buyers (buyers in $B^{3}$ ), but not for employee-buyers (buyers in $B^{1}$ ).

The most important result in the proposition is that in all equilibria of the game, general investment will never be chosen. The basic insight for this is as follows. Remember that when $E^{i}<0$, a general investment is more profitable relative to a 
specific investment for a seller in $S^{i}$. Take $S^{1}$ as an example. If some employer-sellers in $S^{1}$ make a specific investment for buyers in $B^{2} \cup B^{3}$, then definitely $\Delta^{1}<0$ and $E^{1}<0$. In this case, whoever in $S^{1}$ makes a general investment will get a payoff of $m>M / 2$. However, this higher profit is not feasible because each aforementioned seller who made a specific investment for $B^{2} \cup B^{3}$ will have a beneficial unilateral deviation from a specific investment to a general investment so as to make a surplus of $m>M / 2$. What this means is that rationality rules out the possibility of sellers in $S^{1}$ making specific investments for buyers in $B^{2} \cup B^{3}$. Hence $\Delta^{1}=0$ and $E^{1}=0$, and the general investments by any seller in $S^{1}$ becomes unprofitable. A similar argument shows that in turn members in $S^{2}$ and $S^{3}$ will never make general investments.

The proposition also points out two kinds of indeterminacy in the game. First, the person for whom a seller's specific investment is made may vary from one equilibrium to another. For instance, in one equilibrium each employer-seller in $S^{1}$ makes a specific investment for her subordinate-buyer, while another equilibrium differs from the former in that each seller in $S^{1}$ makes a specific investment for some buyer in $B^{1}$ who is not her subordinate. So long as these specific investments are not duplicates, these two equilibria yield the same payoffs to all investing parties. Hence this indeterminacy is inconsequential.

The second type of indeterminacy is somewhat different. It is possible as an equilibrium outcome that an employee-seller (a seller in $S^{3}$ ) does not make any investment, foreseeing that a specific investment for her employee-buyer will be made by some independent seller (seller in $S^{2}$ ). The maximum number of such noninvesting sellers in $S^{3}$ is $\min \left\{\left|S^{3}\right|,\left|S^{2}\right|-\left|B^{2}\right|\right\}$. Taking into account Proposition 3.1 in which some $S^{2}$ 
do not have an investment, we know that the fates of sellers in $S^{2}$ and $S^{3}$ are not as promising as those in $S^{1}$. The ownership of both assets ensures a seller with certainty of successful trade, as evidently sellers in $S^{1}$ all will have trades in all equilibria, but a seller in $S^{2}$ or $S^{3}$ may not have a trade in some equilibrium. In other words, when all equilibria are likely ex ante, absence of ownership cripples the seller with fewer assets and a smaller probability of having trade. In the literature, the absence of ownership is usually thought to lead to inefficiency in investment. The idea that it is conducive to a lower probability of trade is new, as far as we know. ${ }^{13}$

\subsection{Excess Competition among Buyers $(|S|<|B|)$}

In this subsection, we study the opposite case where $|S|<|B|$. In contrast to the previous case, ownership matters but in a way different from Hart and Moore's general insight.

Proposition 4.3. Suppose $|S|<|B|$. Every equilibrium of the game has the following properties:

1. Employer-sellers (sellers in $S^{1}$ ) will each make a general investment.

2. Independent sellers (sellers in $S^{2}$ ) will each make a general investment.

3. Employee-sellers (sellers in $S^{3}$ ) will each make an investment distinctly specific to some employer-buyer in $B^{3}$.

\footnotetext{
${ }^{13}$ Note that while a lower probability of trade is an issue to the agent, it is never an issue to society at large, as far as social welfare is concerned.
} 
It is easy to see that the above description constitutes an equilibrium. Consider the actions of sellers in $S^{1}$ and $S^{2}$. When every other member in $S^{1} \cup S^{2}$ makes a general investment, a member in $S^{1} \cup S^{2}$ can extract a payoff of $m$ by making a general investment. The point is that the number of general investments available for all buyers in $B^{1}$ and $B^{2}$ is $\left|S^{1}\right|+\left|S^{2}\right|$ only. But $\left|B^{1}\right|+\left|B^{2}\right|>\left|S^{1}\right|+\left|S^{2}\right|$. This excess demand drives the equilibrium price up to $m$. The excess demand by $B^{1}$ and $B^{2}$ is the crucial reason that both $S^{1}$ and $S^{2}$ making general investment can constitute an equilibrium here, but cannot in Propositions 4.1 and 4.2 for $|B| \leq|S|$. The general lesson found in Hart and Moore that asset ownership enhances the asset owner's incentive to make more appropriate investments does not hold in this context.

The above points out that the driving force of the inefficiency is that sellers take advantage of the excess competition on the part of buyers. In order to restore efficiency, sellers should refrain from taking advantage of the excess buyer competition. This is precisely why sellers in $S^{3}$ all make efficient, specific investments. In our model, employer-sellers are free to bargain and trade with other buyers. In this sense, they receive no more restrictions than under the no-integration case. The difference, however, is that effectively the excessively competing buyers disappear from the market. Note that sellers in type 3 (integrated) firms now do not possess any asset. In order to have a successful match, they have to look for buyers who have both kinds of assets. That is, they can only bargain and trade with buyers in $B^{3}$, while $B^{1}$ and $B^{2}$ are no longer feasible for these sellers. Now the number of potential buyers for these sellers no longer exceeds the sellers in number. The excess competition among players is therefore eliminated. 
Two remarks are in order here. First, we implicitly assumed that the investment the seller makes is human capital, which is inalienable from the seller. If the investment is physical, then it is even more clearly that type 3 firms are preferable because each seller now cannot use her physical capital, which is a property of the type 3 firm, for any use outside the firm without her boss's approval. The second point is that alleviating the inefficiency problem through ownership arrangements may not be feasible. When the assets $a_{s}$ that sellers need to have access to are inalienable (for instance, $a_{s}$ may be human capital, or a nontransferable license), a change in the ownership of $a_{s}$ is simply impossible. Recall that inefficient decisions are made because there is too little competition among sellers. Thus, one way to correct the incentive is to allow easy entry to the seller market. By allowing enough sellers in the market, sellers would switch from making general investments to making specific investments.

\section{Extensions}

In this section, we want to briefly look at two variants of the model in order to help sharpen our understanding. The first variant is one in which a seller is still productive even if she does not have any investment. The second variant is one in which each seller chooses the investment level, rather than the investment type.

\subsection{Noninvesting Sellers yet Productive}

Let the surplus created by a noninvesting seller and any buyer be the same and equal to $k$. The economically interesting case is where $k<m-c<M-c$. Then it is natural 
to assume that the surplus jointly created by a buyer and a seller with a specific - but not specific to that buyer-investment is also $k$. Using this new set of parameters, we can compute a new bargaining solution that respects the three principles outlined before. It can be shown that when $k$ is small enough $(k / 2<M / 2-c)$, the results we obtained in the last section regarding optimal asset ownership and the role of competition will not be altered at all. However, when $k / 2>M / 2-c$, the story will be quite different. What this condition means is that no investment is more attractive than specific investment as long as the surplus is split equally. It can be shown that, in the case of $|S|>|B|$, in every equilibrium (1) employer-sellers will simply make no investments, while (2) a total number of $\left|B^{2}\right|+\left|B^{3}\right|$ sellers of other types will make specific investments as the last section predicted. In other words, the employersellers no longer plan to make specific investment as the last section predicted. This diversion of incentives does not occur for independent sellers and employee-sellers, however. The reason is that they are not shielded from excess competition among sellers as employer-sellers are. Because $\left|S^{2}\right|+\left|S^{3}\right|>\left|B^{2}\right|+\left|B^{3}\right|$, some sellers from $S^{2}$ and $S^{3}$ are bound to have no buyers. Hence any noninvesting seller in $S^{2}$ and $S^{3}$ will receive a zero payoff from trading with a buyer. But by making a specific investment (provided that no other seller makes the same investment), the seller can secure a positive (net of cost) payoff of $M / 2-c$, which is clearly more preferable. Hence in this case of high value of no investment, competition among the sellers may not be strong enough to induce employer-sellers to make specific investments. The correct incentive is provided only by reducing the assets owned by the sellers. This insight is consistent with the last section in that for the case of $|S|<|B|$, depletion of asset 
ownership will enhance sellers' incentives to make specific investments.

\subsection{Sellers Choosing Investment Levels}

The second variant differs from our main model in the following way. Each seller's decisions are the level of a specific investment, and to whom the investment is specific. This model is more in line with the modeling practise in the property rights theory literature. Denote the investment level as $e$, and the corresponding cost as $c(e)$, which is increasing, strictly convex, twice differentiable, and with $c(0)=0$ and $c^{\prime}(0)=0$. The surplus the seller creates together with a buyer (along with a pair of $a_{s}$ and $a_{b}$ ) is $f(e)$ if the buyer is the one whom the investment is specific for, and is $g(e)$ otherwise. The two functions $f(e)$ and $g(e)$ have the following properties: $f(0)=g(0)=k \geq 0$, $f(e)=k+e, g(e)=k+\alpha e$, where $-\infty<\alpha<1$ (hence $f(e) \geq g(e)$, for all $e$ ). The assumption of linearity of the payoff function in the investment is quite standard, and is without loss of generality; see, for example, Tirole, and Aghion and Tirole (1997) for similar formulations. The interpretation of $\alpha$ is in order. For $0<\alpha<1$, investment increases both the surplus from the prospective relationship with the buyer the investment is targeted for, as well as the surplus from alternative relationships. For $\alpha<0$, investment is so specific that its increase will in fact reduce its value of use in any alternative relationship. This specification is less frequently used in the literature, and is one of the formulations that Rajan and Zingales used in arguing that assigning asset ownership to an investor will reduce his investment incentive. In order to accommodate the specification that at the same time $g^{\prime}(e)<0$ and $g(e)>0$, the possibility that $k>0$ is necessary. To prevent the reduced investment 
incentive studied in the previous subsection due to the high payoff of no investment, $k$ is assumed to be small enough.

A complete analysis of this problem is very involved, mainly because in order to solve the equilibrium of the game one needs to first specify the bargaining outcome for every profile of sellers' investment levels. Here we are content to examine a focal equilibrium in which sellers of the same ownership each make the same level of investment whenever investing. This focal equilibrium can be called semi-symmetric in the sense that an asymmetry may occur such that some sellers of the same ownership may not invest while others do. Consider the case of $|S| \geq|B|$. In this equilibrium, all sellers in $S^{1}$ and $S^{3}$ make investments; only $\left|B^{2}\right|$ sellers in $S^{2}$ make investments; and sellers' investments are of the same level and are not duplicates. ${ }^{14}$ It is easy to see why this can constitute an equilibrium. Given a profile of investments by others as prescribed by this equilibrium, the last seller who is prescribed to invest will have no incentive to deviate by making a duplicate investment. Furthermore, each investing seller must choose a level of nonduplicate investment equal to $e^{*} \equiv \arg \max _{e}\left\{\frac{1}{2} f(e)-c(e)\right\} .^{15}$ In other words, ownership does not play a role in enhancing efficiency; employersellers, independent sellers, and employee-sellers make the same investment whenever investing. The result is invariant for any value of $\alpha$ where $-\infty<\alpha<1$.

The case of $|S|<|B|$ is different. We consider three different specifications here: (1) $k=0$ and $\alpha>1 / 2 ;$ (2) $k=0$ and $0 \leq \alpha \leq 1 / 2$; and (3) $k>0$

\footnotetext{
${ }^{14}$ In order for this to be an equilibrium outcome, we also require that members in $S^{i}$ make investments for $B^{i}$ only, $i=1,2,3$.

${ }^{15}$ Any alternative relationship only will a value of $g\left(e^{*}\right)<f\left(e^{*}\right)$ to the seller and buyer. This renders this outside option unattractive to both the seller and the buyer. Hence, the choice of $e^{*}$ on the part of each investming seller becomes self fulfilling.
} 
and $\alpha<0$. For specification (1), the focal equilibrium has the following features. Employer-sellers and independent sellers each will make a specific investment $\widetilde{e} \equiv$ $\arg \max _{e}\left\{\max \left\{\frac{1}{2} f(e), g(e)\right\}-c(e)\right\}=\arg \max _{e}\{g(e)-c(e)\} .{ }^{16}$ Employee-sellers, however, will only make a specific investment $e^{*} \equiv \arg \max _{e}\left\{\frac{1}{2} f(e)-c(e)\right\}<\widetilde{e}$ as excess outside opportunities are unavailable. Note that $\widetilde{e}$ is closer to the socially optimal investment than $e^{*}$. In this case, in the presence of excess competition on the part of buyers, ownership (of at least one asset) by sellers leads to a more efficient outcome than no ownership at all. For both specifications (2) and (3), the excess opportunities available for employer-sellers and independent sellers are no longer attractive. Hence the focal equilibrium is such that they will make an investment of $e^{*}$, as employeesellers will do. Here ownership does not play any role in enhancing efficiency; all sellers will choose the same investment level. Summing up the results for specifications (1) to (3), we can conclude that, to enhance efficiency, the investor owning more assets is (at least weakly) more preferable. This is in line with Hart and Moore: more assets lead to a greater outside option, hence leading to a greater investment.

A remark contrasting our result and that of Rajan and Zingales is in order. Under our specification (3), i.e., when each seller's value of use in an outside relationship goes down with her investment, we found that assigning ownership of $a_{s}$ or both $a_{s}$ and $a_{b}$ to the seller does not reduce her investment incentive (for both cases of $|S| \geq|B|$ and $|B|<|S|)$. This is in contrast with Rajan and Zingales's prediction of an adverse effect of ownership. The difference is due to the fact that different bargaining solutions are used in the analysis. In our bargaining solution with the outside option principle, an

\footnotetext{
${ }^{16}$ It is because $\left|S^{1}\right|+\left|S^{2}\right|<\left|B^{1}\right|+\left|B^{2}\right|$.
} 
agent's outside option in general does not enhance one's payoff from bargaining unless it reaches a critical level. In the Shapley value, an agent's outside option affects one's bargaining payoff in a positive way. Hence with standard assumptions in Hart and Moore, where outside option increases with investment, Shapley value gives a more positive role of asset ownership in enhancing investment efficiency than the outside option principle does. In Rajan and Zingales, where the outside option decreases with investment, Shapley value gives a more negative role of asset ownership than the outside option principle does.

\section{Concluding Remarks}

This paper studies the optimal asset ownership in a particular setting. While in line with the incomplete contracting approach, the problem discussed is new in that the choice variable is investment type and an explicit market consisting of multiple sellers and buyers is studied. In the section of extensions, we also studied variants of the main model that allow for, respectively, noninvesting sellers to generate a positive surplus and the choice variable to be investment level rather than solely investment type.

One general insight obtained is about the role competition plays in affecting investment incentives. A seller can improve her payoff by two means: (1) to make herself the monopolist of the product wanted by her designated buyer; and (2) to generate competition among the designated buyer and alternative buyers for the product which the seller plans to sell. When the choice variable is investment type, (1) is achieved by choosing specific investment and (2) by choosing general investment. So there is 
a trade-off between these two methods of increasing payoff. In the presence of excess competition among sellers, the second option is so unfavorable that the first option is chosen by every investing seller. In the presence of excess competition among buyers, the second option dominates the first option except for the sellers who do not own any assets. Hence, the trade-off between the two payoff maximizing motives (or between the two investment types) is resolved as a function of the market structure, characterized by the numbers of sellers and buyers as well as the ownership structures. ${ }^{17}$

When the choice variable is investment level, however, the trade-off between the two motives no longer exists. Both motives are fulfilled by the choice of the same specific investment. Whereas the benefit from the first motive (to become a monopolist) is constrained by the necessity of the designated partner, a seller however may enhance her payoff if she can make use of the second motive, which enhances the seller's outside option. Hence, when there are more sellers than buyers, the second motive will lead employer-sellers and independent sellers to choose a greater, more appropriate investment level than employee-sellers will choose.

Two final remarks are in order here. First, the analysis is economized by the use of a bargaining solution, which has the properties (under different competitive scenarios) of: (1) efficiency, (2) Nash bargaining solution, (3) outside option principle, and (4) auction-Walrasian outcome. While we maintain that our bargaining solution is justifiable, we also recognize that different views, especially in which the payoff to the long side's agents is not driven down to zero, are possible. However, the general

\footnotetext{
${ }^{17}$ The above two motives are independently pointed out in a paper by Nicita (1999), which also coins the scenario in which sellers face multiple buyers and have mutiple investment choices as "cross competition."
} 
insight that, when decision variable is investment type, an increase in competition among sellers will make sellers more likely to make specific investment is still valid even if a further relaxation of assumptions is made. The premise of this insight is that, other things being equal, increasing competition among sellers reduces payoff from general investment. Thus so long as a bargaining solution respects this, there must exist a critical number such that regardless of ownership structure sellers will make specific investment when they exceed that number.

Second, our result of the harmful effect of ownership differs from related results in Chiu, de Meza and Lockwood, and Rajan and Zingales in an important dimension. In those papers, the outside market was assumed fixed, independent of the conceived parties. The emphasis was usually on the investment incentives of both parties in a bilateral relationship. That is, the strategic relationship was among parties who would trade/cooperate ex post. Rajan and Zingales, while allowing for a multilateral relationship in which several agents work in a single firm, still retain the feature that these agents know ex ante that they will cooperate ex post. This is not the case here. Here all investors will never benefit from working together as a firm, as in all the other papers cited. Therefore, the strategic interactions occur indirectly through the market. 


\section{References}

[1] Aghion, Philippe and Jean Tirole (1997), "Formal and Real Authority in Organizations," Journal of Political Economy, 105(1): 1-29.

[2] Binmore, Ken, Ariel Rubinstein, and Asher Wolinsky (1986), "The Nash Bargaining Solution in Economic Modelling," Rand Journal of Economics, 17: 176-188.

[3] Binmore, K., A. Shaked and John Sutton (1989), "An Outside Option Experiment," Quarterly Journal of Economics, 104: 753-770.

[4] Bolton, Patrick and Michael D. Whinston (1993), "Incomplete Contracts, Vertical Integration and Supply Assurance," Review of Economic Studies, 60 (1): $121-148$.

[5] Chiu, Y. Stephen (1998), "Noncooperative Bargaining, Hostages, and Optimal Asset Ownership," American Economic Review, 88(4): 882-901.

[6] Dasgupta, S. and Z. Tao (1999), "Bargaining, Bonding, and Partial Ownership," International Economic Review (forthcoming).

[7] de Meza, David and Ben Lockwood (1998a), "Does Asset Ownership Always Motivate Managers? Outside Options and the Property Rights Theory of the Firm," Quarterly Journal of Economics, 113(2): 361-86.

[8] and Matching: the Property Rights Theory of the Theory in Market Equilibrium," mimeo, Universities of Exeter and Warwick. 
[9] Grossman, Sanford J. and Hart, Oliver D. (1986) "The Costs and Benefits of Ownership: A Theory of Vertical and Lateral Integration," Journal of Political Economy, 94(4): 691-719.

[10] Gul, Faruk (1989), "Bargaining Foundations of Shapley Value," Econometrica, $57,81-95$.

[11] _______ (1999), "Efficiency and Immediate Agreement: A Reply to Hart and Levy," Econometrica, 67(4): 913-917.

[12] Hart, Oliver (1995), Firms Contracts and Financial Structures, Oxford University Press: Oxford.

[13] Hart, Oliver and John Moore (1990), "Property Rights and the Nature of the Firm," Journal of Political Economy 98: 1119-1158.

[14] Hart, Sergiu and Mas-Colell, Andreu (1996), "Bargaining and Value," Econometrica, 62(2): 357-80.

[15] Holmstrom, Bengt (1999), "The Firm as a Subeconomy," Journal of Law, Economics, and Organization, 15(1): 74-102.

[16] Holmstrom, Bengt and Jean Tirole (1991), "Transfer Pricing and Organizational Form," Journal of Law, Economics and Organization, 7: 201-228.

[17] Maskin, Eric and Jean Tirole (1999), "Two Remarks on the Property-Rights Literature," Review of Economic Studies, 66: 83-114.

[18] Nash, John F., Jr. (1950) "The Bargaining Problem," Econometrica, 18(2): 15562. 
[19] Nicita, Antonio (1999), "Endogenous Enforcement Devices in Incomplete Contracts: Specific Investments, Market Foreclosure and the Nature of the Firm," mimeo, University of Siena.

[20] Rajan, Raghuram G. and Luigi Zingales (1998), "Power in a Theory of the Firm," Quarterly Journal of Economics, 113(2): 387-432.

[21] Rubinstein, A. (1982), "Perfect Equilibrium in a Bargaining Model," Econometrica 50: 97-109.

[22] Segal, I. and M. Whinston (1998), "Exclusive Contracts and Protection of Investments," mimeo, UC Berkeley and Northwestern.

[23] Shapley, Lloyd S. "A Value for n-Person Games," Contribution to the Theory of Games, Volume II. Princeton, NJ: Princeton University Press, 1953, pp. 307-17.

[24] Sutton, J. (1986), "Non-Cooperative Bargaining Theory: An Introduction," Review of Economic Studies, 53: 705-24.

[25] Tirole, Jean (1999), "Incomplete Contracts: Where Do We Stand?" Econometrica, 67(4): 741-782. 


\section{Appendix A. A sketch of proof of the bargaining solution as a Subgame Perfect Equilibrium Outcome of an Alternating Offer Game.}

Proposition A.1. There exists a subgame perfect equilibrium outcome, which is reached immediately without delay and which approaches our bargaining solution as $\delta$ approaches unity.

Before we describe the equilibrium strategy, some notation is in order. Let $E^{i t}$ be the excess supply of general investment to $B^{i t}$ at substage $t$, and $S_{j}^{i t}\left(B_{j}^{i t}\right)$ be the corresponding $S_{j}^{i}\left(B_{j}^{i}\right)$ still in the market at the beginning of substage $t, i=1,2,3, j=$ $1,2,3$. Let $s_{j k}^{i t}\left(b_{j k}^{i t}\right)$ be the kth seller in $S_{j}^{i t}\left(\right.$ kth buyer in $\left.B_{j}^{i t}\right), i=1,2,3, j=1,2,3$.

We name each player as follows as the game evolves from time $t$ to time $t+1$. Let the kth seller in the sequence $s_{11}^{i, t}, \ldots, s_{1,\left|S_{1}^{i, t}\right|}^{i t}$ who is still in the market at stage $t+1$ be denoted by $s_{1 k}^{i, t+1}$. Let the kth seller in the sequence $s_{21}^{i, t}, \ldots, s_{2,\left|S_{2}^{i, t}\right|}^{i t}, s_{31}^{i, t}, \ldots, s_{3,\left|S_{3}^{i, t}\right|}^{i t}$ who is still in the market at stage $t+1$, has a nonduplicate specific investment, and has a buyer of the specific investment still around at stage $t+1$ be denoted by $s_{2 k}^{i, t+1}$. Let the kth seller in the sequence $s_{31}^{i, t}, \ldots, s_{3,\left|S_{3}^{i, t}\right|}^{i t}$ who is still in the market at stage $t+1$ but with a duplicate specific investment be denoted by $s_{3 k}^{i, t+1}$. Likewise, we can name each buyer as the game evolves. In this order-preserving manner, we can identify each player unambiguously in each stage given any history.

We first define two sets of prices $\widetilde{p}_{j}^{i}$ and $\bar{p}_{j}^{i}$ as follows: 
Prices proposed by sellers in $S_{j}^{i}$

$\begin{array}{llll} & \widetilde{p}_{1}^{i} & \widetilde{p}_{2}^{i} & \widetilde{p}_{3}^{i} \\ E^{i}>0 & 0 & \min \{M-m, M /(1+\delta)\} & 0 \\ E^{i}=0 & m /(1+\delta) & M /(1+\delta) & 0 \\ E^{i}<0 & m & M /(1+\delta) & 0\end{array}$

Prices proposed by buyers in $B_{j}^{i}$

$\begin{array}{llll} & \bar{p}_{1}^{i} & \bar{p}_{2}^{i} & \bar{p}_{3}^{i} \\ E^{i}>0 & 0 & \min \{M-m, M /(1+\delta)\} & 0 \\ E^{i}=0 & \delta m /(1+\delta) & \delta M /(1+\delta) & 0 \\ E^{i}<0 & m & \delta M /(1+\delta) & 0\end{array}$

Table A1

The on-the-equilibrium-path strategy prescription is as follows. P1a (1 and 2) describes what each player does when being a proposer, and P1b (3 to 10) describes what each player does when being a responder and P1a has just been played.

1. When sellers propose, $s_{j k}^{i}$ publicly proposes to sell his investment to anybody at $\widetilde{p}_{j}^{i}, k=1,2, . .,\left|S_{j}^{i}\right| ; i=1,2,3 ; j=1,2,3$.

2. When buyers propose, $b_{j k}^{i}$ proposes $\bar{p}_{j}^{i}$ for any general investment (for $j=1$ ) and for the investment specific to himself (for $j=2,3$ ), $k=1,2, . .,\left|B_{j}^{i}\right| ; i=1,2,3$; 
$j=1,2,3$.

3. $b_{2 k}^{i}$ accepts to buy from the seller who has a specific investment for him where $k=1,2,3, \ldots$, and $i=1,2,3$. Buyer $b_{3 k}^{i}$ accept to buy with equal probability from the sellers who have duplicate specific investments where $k=1,2,3, \ldots$, and $i=1,2,3$.

4. $b_{1 k}^{1}$ first accepts to buy from sellers in $S_{1}^{1}$ in order (i.e., $b_{1 k}^{1}$ buying from $s_{1 k}^{1}$ in $S_{1}^{1}$, etc. $), k=1,2, \ldots$ If $\left|S_{1}^{1}\right|<\left|B_{1}^{1}\right|$, excess buyers each $\left(k=\left|S_{1}^{1}\right|+1,\left|S_{1}^{1}\right|+\right.$ $2, \ldots)$ accept to buy from sellers in $S_{1}^{1}$ independently and randomly with equal probability.

5. $b_{1 k}^{2}$ first accepts to buy from sellers in $S_{1}^{2}$ in order, $k=1,2, \ldots$. If $\left|S_{1}^{2}\right|<\left|B_{1}^{2}\right|$, excess buyers each $\left(k=\left|S_{1}^{2}\right|+1,\left|S_{1}^{1}\right|+2, \ldots\right)$ accept to buy in order from sellers in $S_{1}^{1}$ whose offers are not accepted as prescribed by (4). The remaining excess buyers accept to buy independently and randomly with equal probability from all sellers whose offers are supposed to be accepted as prescribed above by (5).

6. In the same manner as (4) and (5), $b_{1 k}^{3}$ first accept to buy from sellers in $S_{1}^{3}$ in order, $k=1,2, \ldots$. The excess buyers accept to buy in order from sellers in $S_{1}^{2}$ whose offers are not accepted as prescribed in (4) and (5). The remaining buyers accept to buy in order from sellers in $S_{1}^{1}$ whose offers are not accepted as prescribed by (4) and (5). The further remaining buyers accept to buy independently and randomly with equal probability from sellers whose offers are supposed to be accepted as prescribed above by (6). 
7. $s_{j k}^{i}$ accepts to sell to the buyer for whom the seller has a specific investment where $k=1,2,3, \ldots, j=2,3$, and $i=1,2,3$.

8. $s_{1 k}^{3}$ accepts to sell to buyers in $B_{1}^{3}$ in order (i.e., $s_{1 k}^{3}$ sells to $b_{1 k}^{3}$ in $B_{1}^{3}$, etc.), $k=1,2, \ldots$ If $\left|S_{1}^{3}\right|>\left|B_{1}^{3}\right|$, excess sellers $\left(k=\left|B_{1}^{3}\right|+1,\left|B_{1}^{3}\right|+2, \ldots\right)$ accept to sell to all buyers in $B_{1}^{3}$ independently and randomly with equal probability.

9. $s_{1 k}^{2}$ first accepts to sell to buyers in $B_{1}^{2}$ in order, $k=1,2, \ldots$. If $\left|S_{1}^{2}\right|>\left|B_{1}^{2}\right|$, excess sellers $\left(k=\left|S_{1}^{2}\right|+1,\left|S_{1}^{2}\right|+2, \ldots\right)$ each accept to sell in order to buyers in $B_{1}^{1}$ whose offers are not accepted as prescribed by (8). The remaining excess sellers accept to sell independently and randomly with equal probability to all buyers whose offers are supposed to be accepted as prescribed above by (9).

10. In the same manner as (8) and (9), $s_{1 k}^{1}$ first accepts to sell to buyers in $B_{1}^{1}$ in order, $k=1,2,3, \ldots$. The excess sellers accept to sell in order to $B_{1}^{2}$ whose offers are not accepted as prescribed by (9). The remaining sellers accept to sell in order to $B_{1}^{3}$ whose offers are not accepted as prescribed by (8) and (9). The further remaining sellers then accept to sell randomly and independently with equal probability to all buyers whose offers are supposed to be accepted as prescribed above by (10).

It is straightforward to verify the following two lemmas.

Lemma A.2. Given P1a and P1b are played in stage $t$, then $E^{i, t} \gtreqless 0 \Rightarrow E^{i, t+1} \gtreqless 0$.

Lemma A.3. Given P1a and P1b are to be played in stage $t$ and thereafter, the game will end immediately with prices as stipulated in Table A1. 
It is a bit tedious to verify that off the equilibrium path strategies can be constructed in such a way that, while they are best responses, no one can gain by unilateral deviations from P1a or P1b.

Lemma A.4. Given that $P 1$ is played in stages after $t$, there does not exist a beneficial unilateral deviation from P1a or from P1b.

Given Lemmas A2 to A4, Proposition A.1 is immediate. In order to prove that P1a and P1b will be played hereafter, one must show that beneficial unilateral deviations are impossible, (1) when simultaneously all other players follow P1a, and (2) when simultaneously all other players follow P1b. Case (2) is easiest to see and is apparent from Lemma A.3. For case (1), we should consider two types of deviations: lessaggressive and more-aggressive. A less aggressive deviation is one in which upon accepting the offer the payoff to the deviator is less than what P1a and P1b allow him (hence, a lower (greater) asking price when the deviator is a seller (buyer)). A more-aggressive deviation is just the opposite of a less-aggressive deviation. It refers to an asking price greater (lower) than the deviator's asking price as prescribed by P1a when the deviator is a seller (buyer). That a less-aggressive deviation can never be beneficial can be shown easily. Basically, the deviation can be beneficial only when the offer is not accepted, and in the next stage the competition becomes more favorable to the deviator so that he or she can obtain a greater payoff even after discounting. This, however, is impossible, as it will happen only if somebody else makes a strictly dominated decision in stage $t$.

To show that a more aggressive deviation can never be beneficial, one needs to 
stipulate the response strategy prescription - which we call P2 - to any of such unilateral deviations from P1a. P2 is basically the same as P1b, except for the responder who is supposed to accept the deviator's candidate equilibrium proposal prescribed by P1b. Depending on the case, that responder is prescribed either to reject any offer or to accept some other offer. By so doing, this ensures that, if the responder survives the next stage, he will see a competition environment similar to the one in the last stage $\left(E^{i, t+1}=E^{i, t}\right)$. In this case his payoff from stage $t+1$ (given that P1a and P1b are to be played) will be definitely greater than that from accepting the deviating offer in stage $t$. The last thing to show is that no unilateral deviations from P2 will be beneficial given that P1a and P1b are to be played hereafter. This again is indeed true. All of the above altogether shows Proposition A.1.

\section{Appendix B. Proof of Lemma 3.1.}

The first part of the proposition is easy to prove. Result (a) of the second part is trivial. We now show result (b), and proofs of the rest are similar. Suppose the result in (b) is not true, and there exists an allocation in the core such that some seller in $S_{1}^{i}\left(\right.$ say $\left.s^{\prime}\right)$ successfully sells its general investment at $p>0$. Note that $E^{i}>0$ implies excess supply of general investments to $B_{1}^{i}$. Somebody's (say, $s^{\prime \prime}$ ) general investment would be redundant. Now consider a subcoalition which includes every agent but $s^{\prime}$. The worth of this subcoalition would be the same as the grand coalition, denoted by $v$. The reason is that the general investment sold by $s^{\prime}$ is now replaced by that of $s^{\prime \prime}$ and becomes reductant. As the initial allocation assigns a sum of payoffs to members of the subcoalition of $v-p<v$, the subcoalition can then profit by breaking away. It is thus contradictory to the claim that the original allocation is in the core. 
Appendix C. Proof of Proposition 4.2 (where $|S| \geq|B|$ )

The results are developed in several steps. (Recall that the subscript $j=1,2,3$ stands for general investment, nonduplicate specific investment, and duplicate specific investment, respectively.)

Step 1. $\left|S_{3}^{i}\right|=0, i=1,2,3$. Since a duplicate specific investment is sold at zero price $\left(p_{3}^{i}=0, i=1,2,3\right)$, no sellers will make a duplicate investment in equilibrium.

Step 2. $E^{i} \leq 0, i=1,2,3$. Otherwise, some seller $s_{1 k}^{i}$ will have to sell her general investment at a price of zero. This cannot be an equilibrium outcome because, foreseeing this, $s_{1 k}^{i}$ would not have made a general investment in stage 1.

Step 3. $E^{3}=0$. Otherwise, taking into consideration of step 1 and that $E^{3}$ takes an integer value, it must be that $E^{3} \leq-1$, also implying $\Delta^{3} \leq-1$. This means that there exists some seller in $S^{3} \backslash S_{1}^{3} \neq \phi$ who either does not have an investment, or has made a duplicate specific investment, or has made a specific investment whose intended buyer does not exist. This seller could have been made better off by making and selling a general investment at a price of at least $m /(1+\delta)$. Hence a contradiction. Step 4. $E^{2}=0$.

Suppose it is not true. Since $E^{2} \leq 0$ (from step 2) and it takes on an integer value, it must be that $E^{2} \leq-1$. From $E^{3}=0$ (step 3 ), we have $\Delta^{3} \leq 0$. If $\Delta^{3}<0$, then, from the definition of $E^{3}$ and the fact that $E^{3}=0$ (step 3 ), we have $\Delta^{2}+\max \left\{0, \Delta^{1}\right\}>0$. Then $E^{2}=E^{3}$, a contradiction. Hence, $\Delta^{3}=0$, and we have $E^{2}=\max \left\{0, \Delta^{1}\right\}+\Delta^{2}$. Consider any arbitrary seller in $S^{2} \backslash S_{1}^{2}$. Because $S_{3}^{2}=\phi$ (step 1), the seller must be either (i) with no investment or (ii) with an investment uniquely specific to a buyer in $B_{1}^{1}$ or (iii) with an investment uniquely specific to a buyer in $B_{2}^{2}$ or (iv) with an 
investment uniquely specific to a buyer in $B_{2}^{3}$. For cases (i) and (ii), the seller should have made a general investment. Then $\left|S_{1}^{2}\right|$ is increased by one, and consequently $E^{2}$ is increased by one, and still $E^{2} \leq 0$. This means that the deviating seller can sell its general investment at a price of at least $m / 2$. Clearly such a unilateral deviation is beneficial, a contradiction. For case (iii), the seller should have made a general investment. In this case both $\left|S_{1}^{2}\right|$ and $\left|B_{1}^{2}\right|$ will be increased by one, both $\Delta^{1}$ and $\Delta^{2}$ remain unchanged, and consequently $E^{2}$ remains unchanged. In other words, a beneficial unilateral deviation for the seller exists, a contradiction. For case (iv), the seller should have made a general investment. In this case, both $\left|S_{1}^{2}\right|$ and $\left|B_{1}^{3}\right|$ are increased by one. Since $\Delta^{3}$ before the deviation is zero, the above change reduces $\min \left\{0, \Delta^{3}\right\}$ by one as well. Altogether, $E^{2}$ is unchanged and such a deviation becomes beneficial, a contradiction. Hence it must be that $E^{2}=0$.

Step 5. $E^{1}=0$.

Suppose, otherwise, $E^{1}<0$. Since $E^{1}$ must take on an integer value, this means $E^{1} \leq-1$. More importantly, this implies that the general investment made by sellers in $S^{1}$ is sold at a price of $m$. Consider any arbitrary seller in $S^{1} \backslash S_{1}^{1}$. Because $S_{3}^{1}=\phi$, the seller must be either (i) with no investment or (ii) with an investment uniquely special to a buyer in $B_{2}^{1}$ or (iii) with an investment uniquely special to a buyer in $B_{2}^{2}$, or (iv) with an investment uniquely special to a buyer in $B_{2}^{3}$. For case (i), it is easy to show that this seller could be made better off by making a general investment rather than no investment. For cases (ii) to (iv), it is easy to show that the seller can be better off by making a general investment rather than a special investment. (Details similar to that in step 4.) This is contradictory to the assumption that $E^{1}<0$. 
Altogether, this implies that $E^{1}=E^{2}=E^{3}=0$. Now that $p_{1}^{i}$ is $m / 2$, each seller in $S^{i}$ can be made better off by having switched to an investment specific to the buyer who is supposed to purchase the seller's general investment. By so doing, she earns $M / 2$, rather than $m / 2$. What this suggests is that $\left|S_{1}^{i}\right|=\left|B_{1}^{i}\right|=0, i=1,2,3$, and that only specific investments will be made when $|S| \geq|B|$.

Appendix D. Proof of Proposition 4.3. (where $|S|<|B|$ )

The results are developed in several steps. (Recall that the subscript $j=1,2,3$ stands for general investment, nonduplicate specific investment, and duplicate specific investment, respectively.)

Step 1. $\left|S_{3}^{i}\right|=0, i=1,2,3$.

Step 2. $E^{i} \leq 0, i=1,2,3$.

Step 3. $E^{3}=0$.

(The proofs of Steps 1 to 3 are the same as in the last proposition.)

Step 4. $\sum_{i=1}^{3}\left|S_{2}^{i}\right|=\sum_{i=1}^{3}\left|B_{2}^{i}\right|$. Trivial.

Step 5. That $E^{1}=E^{2}=E^{3}=0$ is impossible.

Note that

$$
E^{1}=E^{2} \Leftrightarrow \begin{cases}\text { either } & \Delta^{1}>0 \text { and } \Delta^{2}+\min \left\{0, \Delta^{3}\right\}<0, \\ \text { or } & \Delta^{1}=0 \text { and } \Delta^{2}+\min \left\{0, \Delta^{3}\right\}=0 .\end{cases}
$$

But by definition $\Delta^{1}$ cannot be positive. Hence the above equality implies $\Delta^{1}=0$ and $\Delta^{2}=-\Delta^{3} \geq 0$. Hence we have $\Delta^{1}+\Delta^{2}+\Delta^{3}=0$. Note that, since $S_{3}^{i}=S_{4}^{i}=0$, we have $\left|S_{1}^{i}\right|+\left|S_{2}^{i}\right| \leq\left|S^{i}\right|, i=1,2,3$ and $\left|B_{1}^{i}\right|+\left|B_{2}^{i}\right|=\left|B^{i}\right|, i=1,2,3$. Summing all 
these, we have

$$
\sum_{i=1}^{3}\left(\left|S_{1}^{i}\right|+\left|S_{2}^{i}\right|\right) \leq \sum_{i=1}^{3}\left|S^{i}\right|<\sum_{i=1}^{3}\left|B^{i}\right|=\sum_{i=1}^{3}\left(\left|B_{1}^{i}\right|+\left|B_{2}^{i}\right|\right) .
$$

After rearranging, we have

$$
\sum_{i=1}^{3}\left(\left|B_{2}^{i}\right|-\left|S_{2}^{i}\right|\right)>\sum_{i=1}^{3} \Delta^{i}=0
$$

This is contradictory to Step 4.

Step 6. That $E^{1}<E^{2}=E^{3}=0$ is impossible.

Given $\Delta^{1} \leq 0, E^{2}=E^{3}=0$ implies that $\Delta^{2}=-\Delta^{3} \geq 0$. Given this, $E^{1}<0$ implies $\Delta^{1}<0$. Hence any seller in $S^{1} \backslash S_{1}^{1} \neq \phi$ must be either (i) with no investment or (ii) with a specific investment for a buyer in $\cup_{i=1}^{3} B_{2}^{i}$. In case of (i), the seller could have made a general investment. Then $\Delta^{1}$ is increased by one while $\Delta^{2}$ and $\Delta^{3}$ remain unchanged. Therefore, $E^{1} \leq 0$ and the deviating seller can sell its general investment at a price of at least $m /(1+\delta)$. She is then better off. In case of (ii), the seller could have made a general investment, instead of a specific investment. If his initial specific investment is for a buyer in $B_{2}^{1}$, all $\Delta^{i}$ 's remain unchanged; if in $B_{2}^{2}, \Delta^{1}$ is increased by one, $\Delta^{2}$ is decreased by one, and $\Delta^{3}$ remains unchanged; if in $B_{2}^{3}, \Delta^{1}$ is increased by one, $\Delta^{3}$ is decreased by one, and $\Delta^{2}$ remains unchanged. In all cases, $E^{1}$ remains unchanged. Hence the seller's deviation can lead him to a payoff of $m$, instead of $M / 2$ or 0 . Such a beneficial deviation, however, is impossible in equilibrium, a contradiction. 
Steps 1 to 6 lead to the conclusion that $E^{1}<0, E^{2}<0$, and $E^{3}=0$, implying $p_{1}^{1}=p_{1}^{2}=m$ and $p_{1}^{3}=m / 2$. From here we can easily show that $\left|S_{1}^{1}\right|=\left|S^{1}\right|,\left|S_{1}^{2}\right|=\left|S^{2}\right|$, and $\left|S_{2}^{3}\right|=\left|S^{3}\right|$. This completes the whole proof. 